PROCEEDINGS OF THE AMERICAN MATHEMATICAL SOCIETY

Volume 124, Number 4, April 1996

\title{
THE LANGLANDS CLASSIFICATION FOR GRADED HECKE ALGEBRAS
}

\author{
SAM EVENS \\ (Communicated by Roe Goodman)
}

\begin{abstract}
We establish the Langlands classification for graded Hecke algebras. The proof is analogous to the proof of the classification of highest weight modules for semisimple Lie algebras.
\end{abstract}

\section{INTRODUCTION}

In this paper, we prove that the classification of irreducible representations of a graded Hecke algebra can be reduced to the classification of tempered representations. In particular, any irreducible representation $V$ of a graded Hecke algebra $H$ can be realized as the unique irreducible quotient of $H \otimes_{H_{P}} U$, where $H_{P}$ is the graded Hecke algebra associated to a parabolic subgroup $P$ and $U$ is tempered on the semisimple part of $H_{P}$ and has real part in a certain positive cone on the central part. We call $(P, U)$ Langlands data for $V$ and write $V=J(P, U)$. Moreover, we show $J(P, U) \cong J\left(P^{\prime}, U^{\prime}\right)$ implies $P=P^{\prime}$ and $U \cong U^{\prime}$, so that irreducible representations of $H$ are completely classified by Langlands data. Of course, for reductive real groups, this result is due to Langlands [La], and for reductive p-adic groups, this result is due independently to Silberger and Wallach [Si, BW]. For Hecke algebras associated to p-adic groups with connected center and $q$ not a root of unity, and for graded Hecke algebras associated to p-adic groups, this result can be deduced from the classification of irreducible modules in [KL, Lu1, Lu2]. Thus, our result is really new only for representations of graded Hecke algebras not associated to p-adic groups with connected center (for example, with general unequal parameters). We also note that the case of unramified representations of p-adic groups is equivalent to the case of representations of Hecke algebras when the Hecke algebra parameter $q$ is a prime power, and that the representation theory of graded Hecke algebras is equivalent to the representation theory of Hecke algebras when $q$ is not a root of unity.

Our proof is much simpler than the proofs in the other cases, since the difficulties that arise in the real and p-adic settings do not arise here. In particular, the exponents are just weights, the Jacquet module is given by restriction, and intertwining operators are unnecessary. As a consequence, the proof reduces to a standard lemma of Langlands used in the other cases and some simple considerations analogous to those used in the study of highest weight modules. Essentially

Received by the editors October 5, 1994.

1991 Mathematics Subject Classification. Primary 22E50.

Supported by NSF postgraduate fellowship. 
the same proof works for the case of Hecke algebras. We note that the classification of tempered representations for graded Hecke algebras is likely to be nontrivial, since it does not appear to reduce to the cases associated to p-adic groups, which is known (see [BZ], [KL], [Lu1]).

\section{The graded Hecke Algebra}

1.1. We give the definition of the graded Hecke algebra associated to a root system and a system of parameters as in [Lu2]. Let $(X, R, Y, \check{R}, \Pi)$ be a root system, where as usual $X$ and $Y$ are free finitely generated abelian groups together with a perfect pairing between them, $R \subset X$ and $\check{R} \subset Y$ are finite subsets with a bijection between them, and $\Pi$ is a subset of $R$. $R$ is called the set of roots, $\check{R}$ is called the set of co-roots, and $\Pi=\left\{\alpha_{1}, \ldots, \alpha_{l}\right\}$ is called the set of simple roots. These satisfy well-known properties (cf. [Lu2, 1.1]). There are induced systems of positive roots $R^{+}$and $\check{R^{+}}$. We will assume that the system is reduced so that if $\alpha \in R$, then $2 \alpha \notin R$. Let $W$ be the associated Weyl group, which is generated by simple reflections $s_{\alpha}, \alpha \in \Pi$. A system of parameters $c: \Pi \rightarrow \mathbf{N}$ is a function such that $c\left(\alpha_{i}\right)=c\left(\alpha_{j}\right)$ if there is $w \in W$ such that $w\left(\alpha_{i}\right)=\alpha_{j}$, together with a function $c^{*}:\{\alpha \in \Pi \mid \check{\alpha} \in 2 Y\} \rightarrow \mathbf{N}$.

Let $\mathfrak{t}=X \otimes \mathbf{C}, \mathfrak{t}^{*}=Y \otimes \mathbf{C}$ be the dual vector space, and $\mathfrak{t}_{\mathbf{R}}$ and $\mathfrak{t}_{\mathbf{R}}{ }^{*}$ be the real spans of $X$ and $Y$. For $\lambda \in \mathfrak{t}^{*}, \operatorname{Re}(\lambda)$ has the obvious meaning. Let $\mathcal{A}=S[\mathfrak{t}]$.

1.2. Definition. Given the root system $(X, R, Y, \check{R}, \Pi)$ and the system of parameters $c$, the associated graded Hecke algebra is the tensor product of algebras

$$
H \cong \mathbf{C}[W] \otimes \mathcal{A} \otimes \mathbf{C}[r]
$$

subject to the cross relations

$$
\mathbf{C}[r] \text { is in the center of } \mathrm{H}
$$

and

$$
x \cdot s_{\alpha}-s_{\alpha} \cdot\left(s_{\alpha}(x)\right)=\left(x-s_{\alpha}(x)\right)(g(\alpha)-1),
$$

where $x \in \mathfrak{t}, \alpha \in \Pi$ and

$$
\begin{gathered}
g(\alpha)=1+\frac{2 c(\alpha) r}{\alpha}, \check{\alpha} \notin 2 Y, \\
g(\alpha)=1+\frac{\left(c(\alpha)+c^{*}(\alpha)\right) r}{\alpha}, \check{\alpha} \in 2 Y .
\end{gathered}
$$

We remark that when all $c(\alpha)=1$ and $\check{\alpha} \notin 2 Y$ for all $\alpha$, then the second relation can be written as $x \cdot s_{\alpha}-s_{\alpha} \cdot\left(s_{\alpha}(x)\right)=2 r A_{\alpha}(x)$, where $A_{\alpha}$ is the so-called BGG operator.

1.3. Representation theory. Let $\mathfrak{a}=\{x \in \mathfrak{t} \mid \check{\alpha}(x)=0, \alpha \in \Pi\}$ and $\mathfrak{a}^{*}=$ $\left\{\lambda \in \mathfrak{t}^{*} \mid \lambda(\alpha)=0, \alpha \in \Pi\right\}$. Then $S[\mathfrak{a}]$ is in the center of $H$ and, as an algebra, $H$ decomposes into a tensor product $H \cong H_{s} \otimes S[\mathfrak{a}]$. Here $H_{s}$ is the graded Hecke algebra associated with the root system $\left(X_{s}, R, Y_{s}, \check{R}, \Pi\right)$, where $X_{s}$ and $Y_{s}$ are the subsets of $X$ and $Y$ perpendicular to $\mathfrak{a}^{*}$ and $\mathfrak{a}$ respectively. If $V$ is an irreducible 
$H$-module, then $V \cong V_{s} \otimes \mathbf{C}_{\nu}$, where $V_{s}$ is an irreducible representation of $H_{s}$ and $\mathbf{C}_{\nu}$ is a one-dimensional character of $S[\mathfrak{a}]$.

Let $H$ be a graded Hecke algebra and let $V$ be a finite dimensional $\mathcal{A}$-module. Then the abelian algebra $\mathcal{A}$ induces a generalized weight space decomposition $V=$ $\bigoplus_{\lambda \in \mathfrak{t}^{*}} V_{\lambda}$, where $V_{\lambda}$ is the largest subspace of $V$ on which $x-\lambda(x)$ acts nilpotently for all $x \in \mathfrak{t}$. The set of $\lambda \in \mathfrak{t}^{*}$ such that $V_{\lambda} \neq 0$ are called the weights of $V$. If $V$ is any irreducible $H$-module, then the center of $H$ acts by a character and it follows that $V$ is finite dimensional. In particular, $V$ has a weight space decomposition.

1.4. Definition. Let $V$ be an irreducible $H$-module. Then $V$ is essentially tempered if for every weight $\lambda$ of $V, \operatorname{Re}\left(\lambda\left(x_{i}\right)\right) \leq 0$, where $x_{i}$ is a fundamental coweight, defined by the requirement that $\check{\alpha_{j}}\left(x_{i}\right)=\bar{\delta}_{i j}$ and $\nu\left(x_{i}\right)=0, \nu \in \mathfrak{a}^{*} . V$ is tempered if $V$ is essentially tempered and for every weight $\lambda$ of $V, \operatorname{Re}\left(\lambda \mid \mathfrak{a}_{\mathbf{R}}\right)=0$, where $\mathfrak{a}_{\mathbf{R}}$ is the real span of the set of $x \in X$ perpendicular to the coroots.

1.5. Parabolic subalgebras. Let $\Pi_{P}$ be a subset of $\Pi$ and let $R_{P}$ be the set of roots generated by $\Pi_{P}$ and $\check{R_{P}}$ the set of roots generated by $\check{\alpha}, \alpha \in \Pi_{P}$. Then $\left(X, R_{P}, Y, \check{R}_{P}, \Pi_{P}\right)$ is a root system. Recall that $H$ is the graded Hecke algebra associated to a root system $(X, R, Y, \check{R}, \Pi)$ and system of parameters $c$. Let $H_{P}$ be the graded Hecke algebra associated to the root system $\left(X, R_{P}, Y, \check{R_{P}}, \Pi_{P}\right)$ and the restriction of the parameter set $c$. There is an obvious inclusion $H_{P} \subset H$ given by identifying the abelian algebras $\mathcal{A}$ for the two algebras and using the embedding $W_{P} \rightarrow W$ given by identifying $s_{\alpha} \in W_{P}$ with $s_{\alpha} \in W$, for $\alpha \in \Pi_{P}$. We denote the subalgebra $H_{s}$ described in 1.3 by $H_{M_{s}}$.

For this root system $\left(X, R_{P}, Y, \check{R_{P}}, \pi_{P}\right)$, we have the subspaces $\mathfrak{a}=\mathfrak{a}_{P}$ and $\mathfrak{a}^{*}=\mathfrak{a}_{P}{ }^{*}$ as in 1.3. We denote

$$
\mathfrak{a}^{*+}=\left\{\nu \in \mathfrak{a}^{*} \mid \operatorname{Re}(\nu(\alpha))>0, \alpha \in \Pi-\Pi_{P}\right\} .
$$

If $U$ is a $H_{P}$-module, then $H \otimes_{H_{P}} U$ is the induced $H$-module. If $\tilde{U}$ is a $H_{M_{s}}$ module and $\mathbf{C}_{\nu}$ is a one dimensional representation of $S(\mathfrak{a})$ induced by $\nu \in \mathfrak{a}^{*}$, then we denote by $\tilde{U} \otimes \mathbf{C}_{\nu}$ the corresponding $H_{P}$ module.

\section{The LANGLANDS Classification}

2.1. Theorem. (i) Let $V$ be an irreducible $H$-module. Then $V$ is a quotient of $H \otimes H_{P} U$, where $U=\tilde{U} \otimes \mathbf{C}_{\nu}$ is such that $\tilde{U}$ is a tempered $H_{M_{s}}-$ module and $\nu \in \mathfrak{a}^{*+}$.

(ii) If $U$ is as in (i), then $H \otimes_{H_{P}} U$ has a unique irreducible quotient, which we denote by $J(P, U)$.

(iii) If $J\left(P, \tilde{U} \otimes \mathbf{C}_{\nu}\right) \cong J\left(P^{\prime}, \tilde{U}^{\prime} \otimes \mathbf{C}_{\nu^{\prime}}\right)$, then $P=P^{\prime}, \tilde{U} \cong \tilde{U^{\prime}}$ as $H_{M_{s}}$-modules, and $\nu=\nu^{\prime}$.

2.2. We first require two lemmas due to Langlands. Let $Z$ be a real inner product space of dimension $n$. Let $\left\{\check{\alpha_{1}}, \cdots, \check{\alpha_{n}}\right\}$ be a basis such that $\left(\check{\alpha_{i}}, \check{\alpha_{j}}\right) \leq 0$ whenever $i \neq j$. Let $\left\{\beta_{1}, \cdots, \beta_{n}\right\}$ be a dual basis so that $\left(\check{\alpha}_{i}, \beta_{j}\right)=\delta_{i j}$. For a subset $F$ of $\Pi$, let

$$
S_{F}=\left\{\sum_{j \notin F} c_{j} \beta_{j}-\sum_{i \in F} d_{i} \check{\alpha}_{i} \mid c_{j}>0, j \notin F, d_{i} \geq 0, i \in F\right\} .
$$


2.3. Lemma [BW, IV, 6.11]. Let $x \in Z$. Then $x \in S_{F}$ for a unique subset $F=$ $F(x)$.

If $x \in Z$, then let $x_{0}=\sum_{j \notin F} c_{j} \beta_{j}$, where $x \in S_{F}$ and $x=\sum_{j \notin F} c_{j} \beta_{j}-$ $\sum_{i \in F} d_{i} \check{\alpha}_{i}$. It is clear that if $x_{0}=y_{0}$, then $F(x)=F(y)$. Define a partial ordering on $Z$ by setting $x \geq y$ if $x-y=\sum_{a_{i} \geq 0} a_{i} \check{\alpha}_{i}$.

2.4. Lemma [BW, IV, 6.13]. If $x, y \in Z$ and $x \geq y$, then $x_{0} \geq y_{0}$.

2.5. Proof of 2.1. First we note that we may assume that $R$ generates $\mathfrak{t}$ and hence $\check{R}$ generates $\mathfrak{t}^{*}$, by an easy argument.

2.6. Proof of 2.1(i). Throughout the proof, denote the simple co-roots by $\check{\alpha}_{1}, \cdots, \check{\alpha_{l}}$, and let $\left\{\beta_{1}, \cdots, \beta_{l}\right\}$ be a dual basis to the simple coroots relative to the Killing form as in 2.2. Let $V$ be an irreducible representation of $H$. Let $\lambda$ be a weight of $\mathcal{A}$ on $V$ such that $\operatorname{Re}(\lambda)$ is maximal among real parts of weights of $V$. Let $F=F(\operatorname{Re}(\lambda))$ and let $\Pi_{P}=F, \mathfrak{a}=\mathfrak{a}_{P}$, and $\mathfrak{a}^{*}=\mathfrak{a}_{P}{ }^{*}$. Let $\mathfrak{a}_{s}$ be the elements of $\mathfrak{t}$ perpendicular to $\mathfrak{a}^{*}$. Similarly, let $\mathfrak{a}_{s}{ }^{*}$ be the elements of $\mathfrak{t}^{*}$ perpendicular to $\mathfrak{a}$. We have a splitting $\mathfrak{t}^{*}=\mathfrak{a}^{*} \oplus \mathfrak{a}_{s}{ }^{*}$ and we can identify restriction to $\mathfrak{a}$ (resp. $\mathfrak{a}_{s}$ ) with projection onto $\mathfrak{a}^{*}$ (resp. $\left.\mathfrak{a}_{s}{ }^{*}\right)$ by using the Killing form.

Let $\nu=\left.\lambda\right|_{\mathfrak{a}}$. By construction, $\nu \in \mathfrak{a}^{*+}$. Let $W$ be an irreducible representation of $H_{P}$ appearing in $V$ such that $\mathbf{C}[\mathfrak{a}]$ acts by $\nu$ on $W$. Let $\mu$ be a $\mathbf{C}\left[\mathfrak{a}_{s}\right]$ weight appearing in $W$. Then $\operatorname{Re}(\mu)=-\sum_{i \in F} z_{i} \check{\alpha}_{i}$. To show $W$ is a tempered representation of $H_{M_{s}}$ we must show that all $z_{i} \geq 0$.

Clearly, $\mu+\nu$ is a weight of $V$ and we have

$$
\operatorname{Re}(\mu+\nu)=\sum_{j \notin F} c_{j} \beta_{j}-\sum_{i \in F} z_{i} \check{\alpha}_{i}, c_{j}>0
$$

while

$$
\operatorname{Re}(\lambda)=\sum_{j \notin F} c_{j} \beta_{j}-\sum_{i \in F} d_{i} \check{\alpha}_{i}, c_{j}>0, d_{i} \geq 0
$$

Let $F_{2}=\left\{i \in F \mid z_{i}<0\right\}$ and let $F_{1}=F-F_{2}$. Then $\operatorname{Re}(\mu+\nu) \geq \sum_{j \notin F} c_{j} \beta_{j}-$ $\sum_{i \in F_{1}} z_{i} \check{\alpha}_{i}$. Thus, $\operatorname{Re}(\mu+\nu)_{0} \geq \sum_{j \notin F} c_{j} \beta_{j}=\operatorname{Re}(\lambda)_{0}$. But $\operatorname{Re}(\lambda) \geq \operatorname{Re}(\mu+\nu)$, so $\operatorname{Re}(\lambda)_{0} \geq \operatorname{Re}(\mu+\nu)_{0}$ by Lemma 2.4. Hence, $\operatorname{Re}(\lambda)_{0}=\operatorname{Re}(\mu+\nu)_{0}$, so $F\left(\operatorname{Re}(\lambda)_{0}\right)=$ $F\left(\operatorname{Re}(\mu+\nu)_{0}\right)$. This implies that $F_{2}=\emptyset$, so all $z_{i} \geq 0$.

The inclusion of $H_{P}$-modules, $W \subset V$, induces a nonzero map $\pi: H \otimes_{H_{P}} W \rightarrow V$ given by $\pi(h \otimes w)=h \cdot w$ (by Frobenius reciprocity). Since $V$ is irreducible, $V$ is a quotient of $H \otimes_{H_{P}} W$.

This argument is very similar to the argument given in the proof of the analogous fact for real groups [BW, IV , 3.4]. Note that it follows from the argument that every weight $\lambda$ of $W$ has $F(\operatorname{Re}(\lambda))=F$.

2.7. Proof of 2.1(ii). Note that $U$ is naturally embedded in $H \otimes_{H_{P}} U$. Let

$$
W^{P}=\left\{w \in W \mid w\left(R_{P}^{+}\right) \subset R^{+}\right\}
$$

Then all weights of $\left(H \otimes_{H_{P}} U\right) / U$ are of the form $w^{P}(\lambda)$, where $w^{P} \in W^{P}, w^{P} \neq 1$, and $\lambda$ is weight of $U$ [BM, proof of 6.4]. 
Let $\lambda$ be a weight of $U$, and write $\operatorname{Re}(\lambda)=\sum_{j \notin F} c_{j} \beta_{j}-\sum_{i \in F} d_{i} \check{\alpha}_{i}$ with all $c_{j}>0$ and $d_{i} \geq 0$. Then if $w \in W^{P}$,

$$
R e(w \lambda)=\sum_{j \notin F} c_{j} w \beta_{j}-\sum_{i \in F} d_{i} w \check{\alpha_{i}} .
$$

Define $\rho \in \mathfrak{t}^{*}$ by $\rho(\check{\alpha})=1, \check{\alpha} \in \check{\Pi}$. Since $w: \Pi_{P} \rightarrow R^{+}, \rho\left(w\left(\check{\alpha_{i}}\right)\right) \geq \rho\left(\check{\alpha_{i}}\right), i \in F$. Since $\beta_{j}$ is a fundamental weight, $w\left(\beta_{j}\right) \leq \beta_{j}$ with equality if and only if in a minimal expression for $w$, each simple reflection fixes $\beta_{j}$. For $w \in W^{P}$ and $j \notin F$, this can only happen if $w=1$. It follows that if $w \in W^{P}$ and $w \neq 1$, then $\rho(\operatorname{Re}(w(\lambda)))<\rho(\operatorname{Re}(\lambda))$. Fix a weight $\lambda$ of $U$ such that $\rho(\operatorname{Re}(\lambda))$ is maximal. It follows that $\lambda$ does not occur as a weight of the $\mathcal{A}$-module $\left(H \otimes_{H_{P}} U\right) / U$.

In particular, if a submodule $Z$ of $H \otimes_{H_{P}} U$ contains the weight $\lambda$, then $Z=$ $H \otimes_{H_{P}} U$. Let $I_{\max }$ be the sum of all submodules of $H \otimes_{H_{P}} U$ not containing the weight $\lambda$. It follows by a standard argument that $I_{\max }$ is the maximal proper submodule and $H \otimes_{H_{P}} U$ has a unique irreducible quotient.

2.8. Proof of 2.1(iii). Assume that $\pi: J(P, U) \cong J\left(P^{\prime}, U^{\prime}\right)$. Let $\lambda$ be a weight of $U$ such that $\rho(\operatorname{Re}(\lambda))$ is maximal and let $\lambda^{\prime}$ be a weight of $U^{\prime}$ such that $\rho\left(\operatorname{Re}\left(\lambda^{\prime}\right)\right)$ is maximal. Suppose $F(\operatorname{Re}(\lambda)) \neq F\left(\operatorname{Re}\left(\lambda^{\prime}\right)\right)$. Then it follows that $\lambda^{\prime}$ is not a weight of $U$ (by the remark at the end of 2.6), so by the argument in 2.7, $\rho(\operatorname{Re}(\lambda))<$ $\rho\left(\operatorname{Re}\left(\lambda^{\prime}\right)\right)$. Similarly, it would follow that $\rho(\operatorname{Re}(\lambda))>\rho\left(\operatorname{Re}\left(\lambda^{\prime}\right)\right)$. Hence, $F(\operatorname{Re}(\lambda))=$ $F\left(\operatorname{Re}\left(\lambda^{\prime}\right)\right)$, so $P=P^{\prime}$. Moreover, $\pi(U)=U^{\prime}$, since $U$ (resp. $\left.U^{\prime}\right)$ is the unique $H_{P}$ submodule containing a weight $\lambda_{1}$ such that $\rho(\operatorname{Re}(\lambda))=\rho\left(\operatorname{Re}\left(\lambda_{1}\right)\right)$ (resp. $\left.\rho\left(\operatorname{Re}\left(\lambda^{\prime}\right)\right)=\rho\left(\operatorname{Re}\left(\lambda_{1}\right)\right)\right)$. Hence $U \cong U^{\prime}$ as $H_{P}$ modules.

2.9. Kazhdan and Lusztig also implicitly establish a Langlands classification for representations that are tempered with respect to an arbitrary group homomorphism $V: \mathbf{C}^{*} \rightarrow \mathbf{R}^{*}$. This can be done in our setting also using a linear map $\mathbf{C} \rightarrow \mathbf{R}$. We omit the details.

\section{ACKNOWLEDGMENTS}

The author would like to thank Allen Moy for his encouragement and his suggestions, Cathy Kriloff for correcting certain inaccuracies, and the University of Michigan for its hospitality during the preparation of this paper.

\section{REFERENCES}

[BM] D. Barbasch and A. Moy, Reduction to real infinitesimal character in affine Hecke algebras, Journal Amer. Math. Soc. 6 (1993), 611-635. MR 93k:22015

[BZ] J. Bernstein and A. Zelevinsky, Induced representations of reductive p-adic groups, I. Ann. Sci. E.N.S. 10 (1977), 441-472. MR 58:28310

[BW] A. Borel and N. Wallach, Continuous cohomology, discrete subgroups, and representations of reductive groups, Annals of Math. Studies, no. 94, Princeton University Press, Princeton, NJ, 1980. MR 83c:22018

[KL] D. Kazhdan and G. Lusztig, Proof of the Deligne-Langlands conjecture for Hecke algebras, Invent. Math. 87 (1987), 153-215. MR 88d:11121

[La] R. Langlands, On the classification of irreducible representations of real algebraic groups, Representation theory and harmonic analysis on semisimple Lie groups, Amer. Math. Soc., Providence, RI, 1989, pp. 101-170. MR 91e:22017

[Lu1] G. Lusztig, Cuspidal local systems and graded Hecke algebras I, Inst. Hautes Etudes Sci. Publ. Math. 67 (1988), 145-202. MR 90e:22029 
[Lu2] G. Lusztig, Affine Hecke algebras and their graded version, Journal Amer. Math. Soc. 2 (1989), 599-635. MR 90e:16049

[Si] A. Silberger, The Langlands quotient theorem for p-adic groups, Math. Ann. 236 (1978), 95-104. MR 58:22413

Department of Mathematics, University of Arizona, Tucson, Arizona 85721

E-mail address: evens@math.arizona.edu 